ARTICLE

https://doi.org/10.1038/s41467-019-10333-7

\title{
Reversing the direction of heat flow using quantum correlations
}

\author{
Kaonan Micadei ${ }^{1,2,8}$, John P.S. Peterson ${ }^{3,8}$, Alexandre M. Souza (i) ${ }^{3}$, Roberto S. Sarthour ${ }^{3}$, Ivan S. Oliveira ${ }^{3}$, \\ Gabriel T. Landi ${ }^{4}$, Tiago B. Batalhão ${ }^{5,6}$, Roberto M. Serra (1) ${ }^{1,7} \&$ Eric Lutz ${ }^{2}$
}

Heat spontaneously flows from hot to cold in standard thermodynamics. However, the latter theory presupposes the absence of initial correlations between interacting systems. We here experimentally demonstrate the reversal of heat flow for two quantum correlated spins- $1 / 2$, initially prepared in local thermal states at different effective temperatures, employing a Nuclear Magnetic Resonance setup. We observe a spontaneous energy flow from the cold to the hot system. This process is enabled by a trade off between correlations and entropy that we quantify with information-theoretical quantities. These results highlight the subtle interplay of quantum mechanics, thermodynamics and information theory. They further provide a mechanism to control heat on the microscale.

\footnotetext{
${ }^{1}$ Centro de Ciências Naturais e Humanas, Universidade Federal do ABC, Avenida dos Estados 5001, 09210-580 Santo André, São Paulo, Brazil. ${ }^{2}$ Institute for Theoretical Physics I, University of Stuttgart, D-70550 Stuttgart, Germany. ${ }^{3}$ Centro Brasileiro de Pesquisas Físicas, Rua Dr. Xavier Sigaud 150, $22290-180$ Rio de Janeiro, Rio de Janeiro, Brazil. ${ }^{4}$ Instituto de Física, Universidade de São Paulo, C.P. 66318, 05315-970 São Paulo, SP, Brazil. ${ }^{5}$ Singapore University of Technology and Design, 8 Somapah Road, Singapore 487372, Singapore. ${ }^{6}$ Centre for Quantum Technologies, National University of Singapore, 3 Science Drive 2, Singapore 117543, Singapore. ${ }^{7}$ Department of Physics, University of York, York YO10 5DD, UK. ${ }^{8}$ These authors contributed equally: Kaonan Micadei, John P.S. Peterson. Correspondence and requests for materials should be addressed to R.M.S. (email: serra@ufabc.edu.br)
} 
ccording to Clausius, heat spontaneously flows from a hot body to a cold body ${ }^{1}$. At a phenomenological level, the second law of thermodynamics associates such irreversible behavior with a nonnegative mean entropy production ${ }^{2}$. On the other hand, Boltzmann related it to specific initial conditions of the microscopic dynamics ${ }^{3-5}$. Quantitative experimental confirmation of this conjecture has recently been obtained for a driven classical Brownian particle and for an electrical RC circuit $^{6}$, as well as for a driven quantum $\operatorname{spin}^{7}$, and a driven quantum $\operatorname{dot}^{8}$. These experiments have been accompanied by a surge of theoretical studies on classical and quantum irreversibility $^{9-19}$. It has in particular been shown that a preferred direction of average behavior may be discerned, irrespective of the size of the system ${ }^{20}$.

Initial conditions not only induce irreversible heat flow, they also determine the direction of the heat current. The observation of the average positivity of the entropy production in nature is often explained by the low entropy value of the initial state ${ }^{3}$. This opens the possibility to control or even reverse the direction of heat flow, depending on the initial conditions. In standard thermodynamics, systems are assumed to be uncorrelated before thermal contact. As a result, according to the second law, heat will flow from the hot object to the cold object. However, it has been theoretically suggested that for quantum-correlated local thermal states, heat might flow from the cold to the hot system, thus effectively reversing its direction ${ }^{9-12}$. This phenomenon has been predicted to occur in general multidimensional bipartite systems $s^{9,10}$, including the limiting case of two simple qubits ${ }^{10}$, as well as in multipartite systems ${ }^{11}$.

Here, we report the experimental demonstration of the reversal of heat flow for two initially quantum-correlated qubits (twospin-1/2 systems) prepared in local thermal states at different effective temperatures employing Nuclear Magnetic Resonance (NMR) techniques ${ }^{21,22}$. Allowing thermal contact between the qubits, we track the evolution of the global state with the help of quantum-state tomography ${ }^{21}$. We experimentally determine the energy change of each spin and the variation of their mutual information $^{23}$. For initially correlated systems, we observe a spontaneous heat current from the cold to the hot spin and show that this process is made possible by a decrease of their mutual information. The second law for the isolated two-spin system is therefore verified. However, the standard second law in its local form apparently fails to apply to this situation with initial quantum correlations. We further establish the nonclassicallity of the initial correlation by evaluating its nonzero geometric quantum discord, a measure of quantumness ${ }^{24,25}$. We finally theoretically derive and experimentally investigate an expression for the heat current that reveals the trade off between information and entropy.

\section{Results}

Experimental system. NMR offers an exceptional degree of preparation, control, and measurement of coupled nuclear spin systems $^{21,22}$. It has for this reason become a premier tool for the study of quantum thermodynamics $7,26,27$. In our investigation, we consider two nuclear spins-1/2, in the ${ }^{13} \mathrm{C}$ and ${ }^{1} \mathrm{H}$ nuclei of a ${ }^{13} \mathrm{C}$ labeled $\mathrm{CHCl}_{3}$ liquid sample diluted in Acetone-d6 (Fig. 1b). The sample is placed inside a superconducting magnet that produces a longitudinal static magnetic field (along the positive $z$-axis) and the system is manipulated by time-modulated transverse radiofrequency (rf) fields. We study processes in a time interval of few milliseconds, which is much shorter than any relevant decoherence time of the system (of the order of few seconds) ${ }^{26}$. The dynamics of the combined spins in the sample is thus effectively closed and the total energy is conserved to an excellent approximation. Our aim is to study the heat exchange between the ${ }^{1} \mathrm{H}$ (system A) and ${ }^{13} \mathrm{C}$ (system B) nuclear spins under a partial thermalization process in the presence of initial correlations (Fig. 1a). Employing a sequence of transversal rf-field and longitudinal field-gradient pulses, we prepare an initial state of both nuclear spins (A and B) of the form,

$$
\rho_{\mathrm{AB}}^{0}=\rho_{\mathrm{A}}^{0} \otimes \rho_{\mathrm{B}}^{0}+\chi_{\mathrm{AB}},
$$

where $\chi_{\mathrm{AB}}=\alpha|01\rangle\left\langle 10\left|+\alpha^{*}\right| 10\right\rangle\langle 01|$ is a correlation term and $\rho_{i}^{0}=\exp \left(-\beta_{i} \mathcal{H}_{i}\right) / \mathcal{Z}_{i}$ a thermal state at inverse temperature $\beta_{i}=$ $1 /\left(k_{B} T_{i}\right), i=(\mathrm{A}, \mathrm{B})$, with $k_{B}$ the Boltzmann constant. The state $|0\rangle$ $(|1\rangle)$ represents the ground (excited) eigenstate of the Hamiltonian $\mathcal{H}_{i}$, and $\mathcal{Z}_{i}=\operatorname{Tr}_{i} \exp \left(-\beta_{i} \mathcal{H}_{i}\right)$ is the partition function. The individual nuclear spin Hamiltonian, in a double-rotating frame with the nuclear spins $\left({ }^{1} \mathrm{H}\right.$ and $\left.{ }^{13} \mathrm{C}\right)$ Larmor frequency, may be written as $\mathcal{H}_{i}=h \nu_{0}\left(\mathbf{1}-\sigma_{z}^{i}\right) / 2$, with $v_{0}=1 \mathrm{kHz}$ effectively determined by a nuclei rf-field offset. In Eq. (1), the coupling strength should satisfy $|\alpha| \leq \exp \left[-h \nu_{0}\left(\beta_{\mathrm{A}}+\beta_{\mathrm{B}}\right) / 2\right] /\left(\mathcal{Z}_{\mathrm{A}} \mathcal{Z}_{\mathrm{B}}\right)$ to ensure positivity. We consider two distinct cases: for $\alpha=0$, the spins are initially uncorrelated as assumed in standard thermodynamics, while for $\alpha \neq 0$, the joint state is initially correlated. We note that since $\operatorname{Tr}_{i} \chi_{\mathrm{AB}}=0$, the two spins are locally always in a thermal Gibbs state in both situations. As a result, thermodynamic quantities, such as temperature, internal energy, heat, and entropy, are well defined. A partial thermalization between the qubits is described by the effective (Dzyaloshinskii-Moriya) interaction Hamiltonian, $\mathcal{H}_{\mathrm{AB}}^{\text {eff }}=i(\pi \hbar / 2) J\left(\sigma_{x}^{\mathrm{A}} \sigma_{y}^{B}-\sigma_{y}^{\mathrm{A}} \sigma_{x}^{\mathrm{B}}\right)$, with $J=215.1 \mathrm{~Hz}^{28,29}$, which can be easily realized experimentally. We implement the corresponding evolution operator, $\mathcal{U}_{\tau}=\exp \left(-i \tau \mathcal{H}_{\mathrm{AB}}^{\text {eff }} / \hbar\right)$, by combining free evolutions under the natural hydrogen-carbon scalar coupling and rf-field rotations (Fig. 1c). We further stress that the correlation term should satisfy $\left[\chi_{\mathrm{AB}}, \mathcal{H}_{\mathrm{AB}}^{\text {eff }}\right] \neq 0$ for the heat flow reversal to occur (Supplementary Information).

Thermodynamics. In macroscopic thermodynamics, heat is defined as the energy exchanged between to large bodies at different temperatures ${ }^{2}$. This notion has been successfully extended to small systems initially prepared in thermal Gibbs states ${ }^{30}$, including qubits ${ }^{10}$. Since the interaction Hamiltonian commutes with the total Hamiltonian of the two qubits, $\left[\mathcal{H}_{\mathrm{A}}+H_{\mathrm{B}}, \mathcal{H}_{\mathrm{AB}}^{\text {eff }}\right]=0$, the thermalization operation does not perform any work on the spins ${ }^{31}$. As a result, the mean energy is constant in time and the heat absorbed by one qubit is given by its internal energy variation along the dynamics, $Q_{i}=\Delta E_{i}$, where $E_{i}=\operatorname{Tr}_{i} \rho_{i} \mathcal{H}_{i}$ is the $z$-component of the nuclear spin magnetization. For the combined system, the two heat contributions satisfy ${ }^{9-11}$,

$$
\beta_{\mathrm{A}} Q_{\mathrm{A}}+\beta_{\mathrm{B}} Q_{\mathrm{B}} \geq \Delta I(A: B),
$$

where $\Delta I(A: B)$ is the change of mutual information between $A$ and $B$. The mutual information, defined as $I(A: B)=S_{\mathrm{A}}+S_{\mathrm{B}}-$ $S_{\mathrm{AB}} \geq 0$, is a measure of the total correlations between two systems ${ }^{23}$, where $S_{i}=-\operatorname{Tr}_{i} \rho_{i} \ln \rho_{i}$ is the von Neumann entropy of state $\rho_{i}$. Equation (2) follows from the unitarity of the global dynamics and the Gibbs form of the initial spin states. For initially uncorrelated spins, the initial mutual information is zero. As a result, it can only increase during thermalization, $\Delta I(A: B) \geq$ 0 . Noting that $Q_{\mathrm{A}}+Q_{\mathrm{B}}=0$ for the isolated bipartite system, we find $^{9-11}$,

$$
Q_{\mathrm{B}}\left(\beta_{\mathrm{B}}-\beta_{\mathrm{A}}\right) \geq 0 \quad \text { (uncorrelated). }
$$

Heat hence flows from the hot to the cold spin, $Q_{B}>0$ if $T_{A} \geq$ $T_{\mathrm{B}}$. This is the standard second law. By contrast, for initially 
a

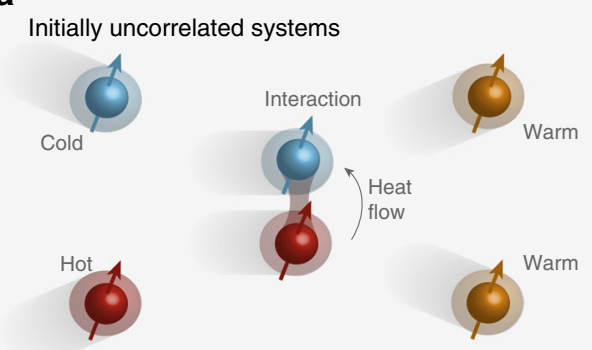

Initially correlated systems

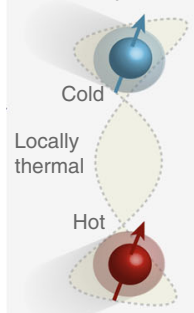

b

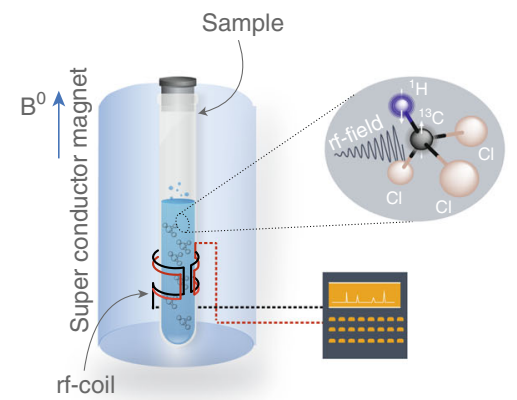

C

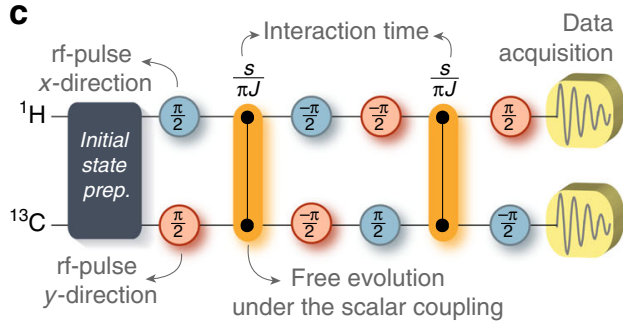

Fig. 1 Schematic of the experimental setup. a Heat flows from the hot to the cold spin (at thermal contact) when both are initially uncorrelated. This corresponds to standard thermodynamic. For initially quantum-correlated spins, heat is spontaneously transferred from the cold to the hot spin. The direction of heat flow is here reversed. $\mathbf{b}$ View of the magnetometer used in our NMR experiment. A superconducting magnet, producing a high-intensity magnetic field $\left(B_{0}\right)$ in the longitudinal direction, is immersed in a thermally shielded vessel in liquid $\mathrm{He}$, surrounded by liquid $\mathrm{N}$ in another vacuum separated chamber. The sample is placed at the center of the magnet within the radio-frequency coil of the probe head inside a 5-mm glass tube. c Experimental pulse sequence for the partial thermalization process. The blue (black) circle represents $x(y)$ rotations by the indicated angle. The orange connections represents a free evolution under the scalar coupling, $\mathcal{H}_{\mathrm{J}}^{\mathrm{HC}}=(\pi \hbar / 2) J \sigma_{z}^{\mathrm{H}} \sigma_{z}^{\mathrm{C}}$, between the ${ }^{1} \mathrm{H}$ and ${ }^{13} \mathrm{C}$ nuclear spins during the time indicated above the symbol. We have performed 22 samplings of the interaction time $\tau$ in the interval 0 to $2.32 \mathrm{~ms}$

correlated qubits, the mutual information may decrease during the thermal contact between the spins. In that situation, we may have $\mathrm{h}^{9-11}$,

$$
Q_{\mathrm{B}}\left(\beta_{\mathrm{B}}-\beta_{\mathrm{A}}\right) \leq 0 \quad \text { (correlated). }
$$

Heat flows in this case from the cold to the hot qubit: the energy current is reversed. We quantitatively characterize the occurrence of such reversal by computing the heat flow at any time $\tau$, obtaining (see the Methods section),

$$
\Delta \beta Q_{\mathrm{B}}=\Delta I(A: B)+S\left(\rho_{\mathrm{A}}^{\tau} \| \rho_{\mathrm{A}}^{0}\right)+S\left(\rho_{\mathrm{B}}^{\tau} \| \rho_{\mathrm{B}}^{0}\right),
$$

where $\Delta \beta=\beta_{\mathrm{B}}-\beta_{A} \geq 0$ and $S\left(\rho_{i}^{\tau} \| \rho_{i}\right)=\operatorname{Tr}_{i} \rho_{i}^{\tau}\left(\ln \rho_{i}^{\tau}-\ln \rho_{i}\right) \geq$ 0 denotes the relative entropy ${ }^{23}$ between the evolved $\rho_{\mathrm{A}(\mathrm{B})}^{\tau}=$ $\operatorname{Tr}_{\mathrm{B}(\mathrm{A})} \mathcal{U}_{\tau} \rho_{\mathrm{AB}}^{0} \mathcal{U}_{\tau}^{\dagger}$ and the initial $\rho_{\mathrm{A}(\mathrm{B})}^{0}$ reduced states. The latter quantifies the entropic distance between the state at time $\tau$ and the initial thermal state. It can be interpreted as the entropy production associated with the irreversible heat transfer, or to the entropy produced during the ensuing relaxation to the initial thermal state ${ }^{32,33}$. According to Eq. (5), the direction of the energy current is therefore reversed whenever the decrease of mutual information compensates the entropy production. The fact that initial correlations may be used to decrease entropy has first been emphasized by Lloyd ${ }^{34}$ and further investigated in refs. ${ }^{35,36}$. Heat flow reversal has recently been interpreted as a refrigeration process driven by the work potential stored in the correlations ${ }^{12}$. In that context, Eq. (5) can be seen as a generalized Clausius inequality due to the positivity of the relative entropies. The coefficient of performance of such a refrigeration is then at most that of Carnot ${ }^{12}$.

In our experiment, we prepare the two-qubit system in an initial state of the form (1) with effective spin temperatures $\beta_{\mathrm{A}}^{-1}=$ $4.66 \pm 0.13 \mathrm{peV}\left(\beta_{\mathrm{A}}^{-1}=4.30 \pm 0.11 \mathrm{peV}\right)$ and $\beta_{\mathrm{B}}^{-1}=3.31 \pm 0.08$ $\operatorname{peV}\left(\beta_{\mathrm{B}}^{-1}=3.66 \pm 0.09 \mathrm{peV}\right)$ for the uncorrelated (correlated) case $\alpha=0.00 \pm 0.01(\alpha=-0.19 \pm 0.01)$ (Supplementary Information). The value of $\alpha$ was chosen to maximize the current reversal. In order to quantify the quantumness of the initial correlation in the correlated case, we consider the normalized geometric discord, defined as $D_{\mathrm{g}}=\min _{\psi \in \mathcal{C}} 2\|\rho-\psi\|^{2}$, where $\mathcal{C}$ is the set of all states classically correlated ${ }^{24,25}$. The geometric discord has a simple closed-form expression for two qubits that can be directly evaluated from the measured QST data (Supplementary Information). We find the nonzero value $D_{\mathrm{g}}=$ $0.14 \pm 0.01$ for the initially correlated state prepared in the experiment.

We experimentally reconstruct the global two-qubit density operator using quantum-state tomography ${ }^{21}$ and evaluate the changes of internal energies of each qubit, of mutual information, and of geometric quantum discord during thermal contact (Fig. 2a-f). We observe the standard second law in the absence of initial correlations $(\alpha \simeq 0)$, i.e., the hot qubit $A$ cools down, $Q_{\mathrm{A}}<0$, while the cold qubit $B$ heats up, $Q_{\mathrm{B}}>0$ (circles symbols in Fig. $2 a, b)$. At the same time, the mutual information and the geometric quantum discord increase, as correlations build up following the thermal interaction (circles symbols in Fig. 2c, d). The situation changes dramatically in the presence of initial quantum correlations $(\alpha \neq 0)$ : the energy current is here reversed in the time interval $0<\tau<2.1 \mathrm{~ms}$, as heat flows from the cold to the hot spin, $Q_{\mathrm{A}}=-Q_{\mathrm{B}}>0$ (squares symbols in Fig. 2a, b). This reversal is accompanied by a decrease of mutual information and geometric quantum discord (squares symbols in Fig. 2c, d). In this case, quantum correlations are converted into energy and used to switch the direction of the heat flow, in an apparent violation of the second law. Correlations reach their minimum at around $\tau \approx 1.05 \mathrm{~ms}$, after which they build up again. Once they have passed their initial value at $\tau \approx 2.1 \mathrm{~ms}$, energy is transferred in the expected direction, from hot to cold. In all cases, we obtain good agreement between experimental data (symbols) and 

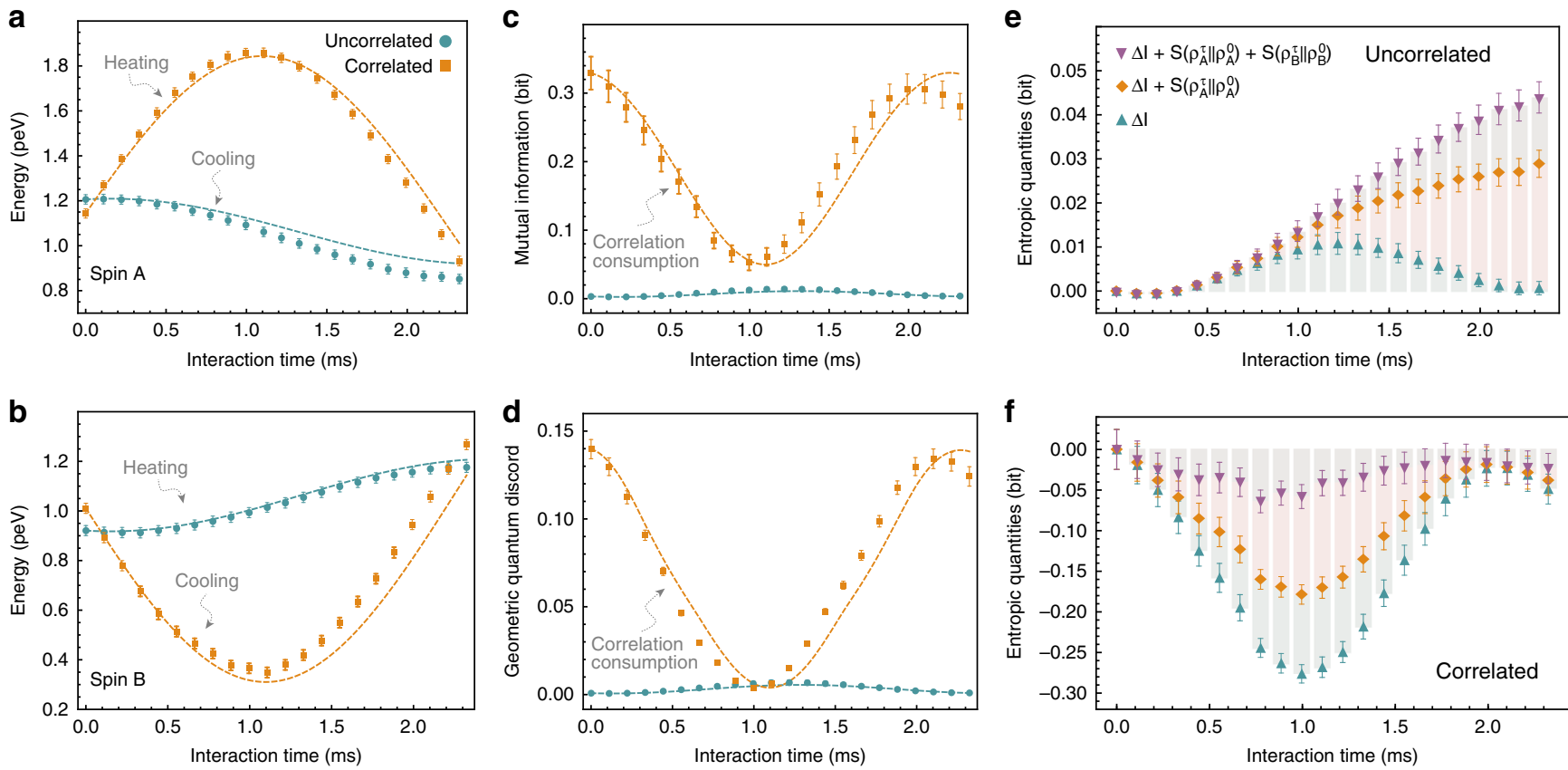

Fig. 2 Dynamics of heat, correlations, and entropic quantities. a Internal energy of qubit A along the partial thermalization process. $\mathbf{b}$ Internal energy of qubit B. In the absence of initial correlations, the hot qubit A cools down and the cold qubit B heats up (cyan circles in panel $\mathbf{a}$ and $\mathbf{b}$ ). By contrast, in the presence of initial quantum correlations, the heat current is reversed as the hot qubit $A$ gains and the cold qubit $B$ loses energy (orange squares in panel $\mathbf{a}$ and $\mathbf{b}$ ). This reversal is made possible by a decrease of the mutual information $\mathbf{c}$ and of the geometric quantum discord $\mathbf{d}$. Different entropic contributions to the heat current (5) in the uncorrelated $\mathbf{e}$ and uncorrelated $\mathbf{f}$ case. Reversal occurs when the negative variation of the mutual information, $\Delta I(A: B)$, compensates the positive entropy productions, $S\left(\rho_{\mathrm{A}}^{\tau} \| \rho_{\mathrm{A}}\right)$ and $S\left(\rho_{\mathrm{B}}^{\tau} \| \rho_{\mathrm{B}}\right)$, of the respective qubits. The symbols represent experimental data and the dashed lines are numerical simulations. Error bars were estimated by a Monte Carlo sampling from the standard deviation of the measured data (Supplementary Information)

theoretical simulations (dashed lines). Small discrepancies seen as time increases are mainly due to inhomogeneities in the control fields.

The experimental investigation of Eq. (5) as a function of the thermalization time is presented in Fig. 2e, f. While the relative entropies steadily grow in the absence of initial correlations, they exhibit an increase up to $1.05 \mathrm{~ms}$ followed by a decrease in presence of initial correlations. The latter behavior reflects the pattern of the qubits already seen in Fig. $2 a, b$, for the average energies. We note, in addition, a positive variation of the mutual information in the uncorrelated case and a large negative variation in the correlated case. The latter offsets the increase of the relative entropies and enables the reversal of the heat current. These findings provide direct experimental evidence for the trading of quantum mutual information and entropy production.

\section{Discussion}

We have observed the reversal of the energy flow between two quantum-correlated qubits with different effective temperatures, associated with the respective populations of the two levels. Such effect has been predicted to exist in general multidimensional systems ${ }^{9-11}$. By revealing the fundamental influence of initial quantum correlations on the direction of thermodynamic processes, which Eddington has called the arrow of time ${ }^{37}$, our experiment highlights the subtle interplay of quantum mechanics, thermodynamics, and information theory. Initial conditions thus not only break the time-reversal symmetry of the otherwise reversible dynamics, they also determine the direction of a process. Our findings further emphasize the limitations of the standard local formulation of the second law for initially correlated systems and offers at the same time a mechanism to control heat on the microscale. They additionally establish that the arrow of time is not an absolute but a relative concept that depends on the choice of initial conditions. We have observed the reversal of the energy current for the case of two spins which never fully thermalize due to their finite size. However, their dynamics is identical to that of a thermalization map during the duration of the experiment (Methods), a process we have labeled partial thermalization for this reason. Furthermore, numerical simulations show that reversals may also occur for a spin interacting with larger spin environments which induce thermalization (Supplementary Information). Hence, an anomalous heat current does not seem to be restricted to extremely microscopic systems. The precise scaling of this effect with the system size is an interesting subject for future experimental and theoretical investigations. Our results on the reversal of the thermodynamic arrow of time might also have stimulating consequences on the cosmological arrow of time $\mathrm{s}^{34}$.

\section{Methods}

Experimental setup. The liquid sample consist of $50 \mathrm{mg}$ of $99 \%{ }^{13} \mathrm{C}$-labeled $\mathrm{CHCl}_{3}$ (Chloroform) diluted in $0.7 \mathrm{ml}$ of $99.9 \%$ deutered Acetone-d6, in a flame sealed Wildmad LabGlass 5 -mm tube. Experiments were carried out in a Varian $500 \mathrm{MHz}$ Spectrometer employing a double-resonance probe head equipped with a magnetic field-gradient coil. The sample is very diluted, such that the intermolecular interaction can be neglected, in this way the sample can be regarded as a set of identically prepared pairs of spin-1/2 systems. The superconducting magnet (illustrated in Fig. 1b) inside of the magnetometer produces a strong intensity longitudinal static magnetic field (whose direction is taken to be along the positive $z$ axes), $B_{0} \approx 11.75 \mathrm{~T}$. Under this filed, the ${ }^{1} \mathrm{H}$ and ${ }^{13} \mathrm{C}$ Larmor frequencies are about $500 \mathrm{MHz}$ and $125 \mathrm{MHz}$, respectively. The state of the nuclear spins are controlled by time-modulated rf-field pulses in the transverse ( $x$ and $y)$ direction and longitudinal field gradients.

Spin-lattice relaxation times, measured by the inversion recovery pulse sequence, are $\left(\mathcal{I}_{1}^{\mathrm{H}}, \mathcal{I}_{1}^{\mathrm{C}}\right)=(7.42,11.31) \mathrm{s}$. Transverse relaxations, obtained by the Carr-Purcell-Meiboom-Gill (CPMG) pulse sequence, have characteristic times $\left(\mathcal{I}_{2}^{* \mathrm{H}}, \mathcal{I}_{2}^{* \mathrm{C}}\right)=(1.11,0.30) \mathrm{s}$. The total experimental running time, to implement 
the partial spin thermalization, is about $2.32 \mathrm{~ms}$, which is considerably smaller than the spin-lattice relaxation and therefore decoherence can be disregarded.

Heat current between initially correlated systems. We here derive the expression (5) for the heat flow between initially correlated systems A and B. For an initial thermal state $\rho_{i}^{0}=\exp \left(-\beta_{i} \mathcal{H}_{i}\right) / \mathcal{Z}_{i},(i=A, B)$, the relative entropy between the evolved $\rho_{\mathrm{A}(\mathrm{B})}^{\tau}=\operatorname{Tr}_{\mathrm{B}(\mathrm{A})} \mathcal{U}_{\tau} \rho_{\mathrm{AB}}^{0} \mathcal{U}_{\tau}^{\dagger}$ and the initial $\rho_{i}^{0}$ marginal states reads,

$$
S\left(\rho_{i}^{\tau} \| \rho_{i}^{0}\right)=-S\left(\rho_{i}^{\tau}\right)+\beta_{i} \operatorname{Tr}_{i}\left(\rho_{i}^{\tau} \mathcal{H}_{i}\right)+\ln \mathcal{Z}_{i},
$$

where $S\left(\rho_{i}^{\tau}\right)$ is the von Neumann entropy of the state $\rho_{i}^{\tau}$. Noting that as $S\left(\rho_{i}^{0} \| \rho_{i}^{0}\right)=0$, one can write,

$$
S\left(\rho_{i}^{\tau} \| \rho_{i}^{0}\right)=S\left(\rho_{i}^{\tau} \| \rho_{i}^{0}\right)-S\left(\rho_{i}^{0} \| \rho_{i}^{0}\right)=-\Delta S_{i}+\beta_{i} \Delta E_{i},
$$

with the variation in the von Neumann entropy, given by $\Delta S_{i}=S\left(\rho_{i}^{\tau}\right)-S\left(\rho_{i}^{0}\right)$ and the internal energy change of the $i$-th subsystem defined as $\Delta E_{i}=$ $\operatorname{Tr}_{i} \rho_{i}^{\tau} \mathcal{H}_{i}-\operatorname{Tr}_{i} \rho_{i}^{0} \mathcal{H}_{i}$. Energy conservation for the combined isolated system (AB) further implies that $\Delta E_{\mathrm{A}}=-\Delta E_{\mathrm{B}}=Q_{\mathrm{B}}$, for constant interaction energy. As a result, we obtain,

$$
Q_{\mathrm{B}} \Delta \beta=\Delta I(A: B)+S\left(\rho_{\mathrm{A}}^{\tau} \| \rho_{\mathrm{A}}^{0}\right)+S\left(\rho_{\mathrm{B}}^{\tau} \| \rho_{\mathrm{B}}^{0}\right),
$$

where $\Delta \beta=\beta_{\mathrm{A}}-\beta_{\mathrm{B}}$ is the inverse temperature difference and $\Delta S_{\mathrm{A}}+\Delta S_{\mathrm{B}}=\Delta I(A$ : $B$ ) holds since the combined system $(\mathrm{AB})$ is isolated.

Partial thermalization. We further show that the spin dynamics is given by a thermalization map during the duration of the experiment. From the local point of view of each individual nuclear spin (when the spins are initially uncorrelated), the evolution operator $\mathcal{U}_{\tau}=\exp \left(-i \tau \mathcal{H}_{\mathrm{AB}}^{\text {eff }} / \hbar\right)$, with $\tau \in\left[0,(2 J)^{-1}\right]$, has the effect of a linear non-unitary map $\mathcal{E}\left(\rho_{i}\right)=\operatorname{Tr}_{k}\left(\mathcal{U}_{\tau} \rho_{\mathrm{A}}^{0} \otimes \rho_{\mathrm{B}}^{0} \mathcal{U}_{\tau}^{\dagger}\right)$ on the spin $i$, which can be represented as,

$$
\mathcal{E}\left(\rho_{i}\right)=\sum_{j=1}^{4} K_{j} \rho_{i}^{0} K_{j}^{\dagger}
$$

where $i=A, k=B$ or $i=B, k=A$. The Kraus corresponding operators $K_{j}$, with $j=$ $(1, \ldots, 4)$, are given by,

$$
\begin{gathered}
K_{1}=\sqrt{1-p}\left[\begin{array}{cc}
1 & 0 \\
0 & \cos (\pi J \tau)
\end{array}\right], \\
K_{2}=\sqrt{1-p}\left[\begin{array}{cc}
0 & \sin (\pi J \tau) \\
0 & 0
\end{array}\right], \\
K_{3}=\sqrt{p}\left[\begin{array}{cc}
\cos (\pi J \tau) & 0 \\
0 & 1
\end{array}\right], \\
K_{4}=\sqrt{p}\left[\begin{array}{cc}
0 & 0 \\
-\sin (\pi J \tau) & 0
\end{array}\right],
\end{gathered}
$$

where $p$ is the population of the excited state in the other spin at time $\tau$. In the time window of the experiment, $\pi J \tau$ varies between zero and $\pi / 2$. In this way, the transformation (9) is equivalent to the generalized amplitude damping ${ }^{38}$ which is the Kraus map for the thermalization of a single spin-1/2 system. Therefore, from the local point of view and in the absence of initial correlations, the interaction implemented in the experiment is indistinguishable from a thermalization map for $\tau \in\left[0,(2 J)^{-1}\right]$.

\section{Data availability}

The datasets generated during and/or analysed during the current study are available from serra@ufabc.edu.br on reasonable request.

Received: 15 January 2018 Accepted: 18 April 2019

Published online: 05 June 2019

\section{References}

1. Clausius, R. The Mechanical Theory of Heat (MacMillan, London, 1879).

2. Callen, H. B. Thermodynamics and an Introduction to Thermostatistics (Wiley, New York, 1985).

3. Boltzmann, L. On the relation of a general mechanical theorem to the second law of thermodynamics. Sitz. Akad. Wiss. Vienna, part II 75, 67-73 (1877).

4. Lebowitz, J. L. Boltzmann's entropy and time's arrow. Phys. Today 46, 32-38 (1993).

5. Zeh, H. D. The Physical Basis of the Direction of Time (Springer, Berlin, 2007).

6. Andrieux, D. et al. Entropy production and time asymmetry in nonequilibrium fluctuations. Phys. Rev. Lett. 98, 150601 (2007).
7. Batalhão, T. M. et al. Irreversibility and the arrow of time in a quenched quantum system. Phys. Rev. Lett. 115, 190601 (2015).

8. Hofmann, A. et al. Heat dissipation and fluctuations in a driven quantum dot. Phys. Status Solidi B 254, 1600546 (2017).

9. Partovi, M. H. Entanglement versus stosszahlansatz: disappearance of the thermodynamic arrow in a high-correlation environment. Phys. Rev. E 77, 021110 (2008)

10. Jevtic, S., Jennings, D. \& Rudolph, T. Maximally and minimally correlated states attainable within a closed evolving system. Phys. Rev. Lett. 108, 110403 (2012).

11. Jennings, D. \& Rudolph, T. Entanglement and the thermodynamic arrow of time. Phys. Rev. E 81, 061130 (2010).

12. Bera, M. N., Riera, A., Lewenstein, M. \& Winter, A. Generalized laws of thermodynamics in the presence of correlations. Nat. Commun. 8, 2180 (2017).

13. Feng, E. H. \& Crooks, G. E. The length of time's arrow. Phys. Rev. Lett. 101, 090602 (2008).

14. Maccone, L. Quantum solution to the arrow-of-time dilemma. Phys. Rev. Lett. 103, 080401 (2009).

15. Parrondo, J. M. R., den Broeck, C. W. \& Kawai, R. Entropy production and the arrow of time. New J. Phys. 11, 073008 (2009).

16. Jarzynski, C. Equalities and inequalities: irreversibility and the second law of thermodynamics at the nanoscale. Annu. Rev. Condens. Matter Phys. 2, 329-351 (2011).

17. Roldán, É., Neri, I., Dörpinghaus, I. M., Meyr, H. \& Jülicher, F. Decision making in the arrow of time. Phys. Rev. Lett. 115, 250602 (2015).

18. Vedral, $\mathrm{V}$. The arrow of time and correlations in quantum physics. arXiv:1605.00926.

19. Dressel, J., Chantasri, A., Jordan, A. N. \& Korotkov, A. N. Arrow of Time for Continuous Quantum Measurement. Phys. Rev. Lett. 119, 220507 (2017).

20. Campisi, M. \& Hänggi, P. Fluctuation, dissipation and the arrow of time. Entropy 13, 2024 (2011).

21. Oliveira, I., Sarthour, R. S., Bonagamba, T., Azevedo, E. \& Freitas, J. C. C. NMR Quantum Information Processing (Elsevier, Amsterdam, 2007).

22. Vandersypen, L. M. K. \& Chuang, I. L. NMR techniques for quantum control and computation. Rev. Mod. Phys. 76, 1037-1069 (2005).

23. Nielsen, M. A. \& Chuang, I. L. Quantum Computation and Quantum Information (Cambridge University Press, Cambridge, 2010).

24. Dakić, B., Vedral, V. \& Brukner, Č. Necessary and sufficient condition for nonzero quantum discord. Phys. Rev. Lett. 105, 190502 (2010).

25. Girolami, D. \& Adesso, G. Observable measure of bipartite quantum correlations. Phys. Rev. Lett. 108, 150403 (2012).

26. Batalhão, T. B. et al. Experimental reconstruction of work distribution and study of fluctuation relations in a closed quantum system. Phys. Rev. Lett. 113, 140601 (2014)

27. Camati, P. A. et al. Experimental rectification of entropy production by Maxwell's demon in a quantum system. Phys. Rev. Lett. 117, 240502 (2016).

28. Scarani, V., Ziman, M., Štelmachovič, P., Gisin, N. \& Bužek, V. Thermalizing quantum machines: dissipation and entanglement. Phys. Rev. Lett. 88, 097905 (2002).

29. Ziman, M. et al. Diluting quantum information: an analysis of information transfer in system-reservoir interactions. Phys. Rev. A. 65, 042105 (2002).

30. Jarzynski, C. \& Wójcik, D. K. Classical and quantum fluctuation theorems for heat exchange. Phys. Rev. Lett. 92, 230602 (2004).

31. Barra, F. The thermodynamic cost of driving quantum systems by their boundaries. Sci. Rep. 5, 14873 (2015).

32. Deffner, S. \& Lutz, E. Generalized Clausius inequality for nonequilibrium quantum processes. Phys. Rev. Lett. 105, 170402 (2010).

33. Deffner, S. \& Lutz, E. Nonequilibrium entropy production for open quantum systems. Phys. Rev. Lett. 107, 140404 (2011).

34. Lloyd, S. Use of mutual information to decrease entropy: implications for the second law of thermodynamics. Phys. Rev. A. 39, 5378 (1989).

35. Sagawa, T. \& Ueda, M. Fluctuation theorem with information exchange: role of correlations in stochastic thermodynamics. Phys. Rev. Lett. 109, 180602 (2012).

36. Koski, J. V., Maisi, V. F., Sagawa, T. \& Pekola, J. P. Experimental observation of the role of mutual information in the nonequilibrium dynamics of a Maxwell demon. Phys. Rev. Lett. 113, 030601 (2014).

37. Eddington, A. S. The Nature of the Physical World (Macmillan, London, 1928).

38. Srikanth, R. \& Banerjee, S. Squeezed generalized amplitude damping channel. Phys. Rev. A. 77, 012318 (2008).

\section{Acknowledgements}

We acknowledge financial support from UFABC, CNPq, CAPES, FAPERJ, and FAPESP (grants 2016/08721-7 and 2018/12813-0). R.M.S. gratefully acknowledges financial support from the Royal Society through the Newton Advanced Fellowship 
scheme (Grant no. NA140436) and the technical support from the Multiuser Experimental Facilities of UFABC. A.M.S. acknowledges support from the Brazilian agency FAPERJ (203.166/2017). K. M. acknowledges CAPES and DAAD for financial support. E.L. acknowledges support from the German Science Foundation (DFG) (Grant no. FOR 2724). This research was performed as part of the Brazilian National Institute of Science and Technology for Quantum Information (INCT-IQ).

\section{Author contributions}

K.M., J.P.S.P., T.B.B., R.S.S., and R.M.S. designed the experiment, J.P.S.P., A.M.S., R.S.S., I.S.O., and R.M.S. performed the experiment, K.M., G.T.L., and E.L. contributed to the theory. All authors contributed to analyzing the data and writing the paper.

\section{Additional information}

Supplementary Information accompanies this paper at https://doi.org/10.1038/s41467019-10333-7.

Competing interests: The authors declare no competing interests.

Reprints and permission information is available online at http://npg.nature.com/ reprintsandpermissions/
Journal peer review information: Nature Communications thanks the anonymous reviewer(s) for their contribution to the peer review of this work.

Publisher's note: Springer Nature remains neutral with regard to jurisdictional claims in published maps and institutional affiliations.

\section{(c) (i)}

Open Access This article is licensed under a Creative Commons Attribution 4.0 International License, which permits use, sharing, adaptation, distribution and reproduction in any medium or format, as long as you give appropriate credit to the original author(s) and the source, provide a link to the Creative Commons license, and indicate if changes were made. The images or other third party material in this article are included in the article's Creative Commons license, unless indicated otherwise in a credit line to the material. If material is not included in the article's Creative Commons license and your intended use is not permitted by statutory regulation or exceeds the permitted use, you will need to obtain permission directly from the copyright holder. To view a copy of this license, visit http://creativecommons.org/ licenses/by/4.0/.

(C) The Author(s) 2019 